\title{
A new mathematical model of peristaltic flow on esophageal bolus transport
}

\begin{abstract}
Ethem Toklu
Department of Mechanical Engineering, Duzce University, 81000 Duzce, Turkey. E-mail: ethemtoklu@duzce.edu.tr.

Accepted 22 November, 2011

A new mathematical model is developed for peristaltic transport in the esophagus. Manometric measurements of luminal pressure have been obtained in the esophagus and interpreted both biological and mechanical point of view. Biological data analysis pointed out that pressure distribution has a polynomial form along the esophagus. However, it has been considered as sinusoidal in calculations by scientists. Therefore, a mathematical result does not possibly fit same form with biological data. The developed model is calculated successfully with pressure distribution along the esophagus. The model is applied to a single contraction wave of normal swallowing in the esophagus. Analytical calculation from the new model on pressure distribution is in qualitative agreement with experimental observations.
\end{abstract}

Key words: Esophagus, bolus transport, peristalsis, peristaltic flow, single wave propagation, polynomial pressure distribution.

\section{INTRODUCTION}

The process of swallowing begins with chewing, mashing and mixing of food in the oral cavity; the complex structural motions within the pharynx that force the food bolus rapidly to the esophagus then follow; and the process ends with the transport of the bolus to the stomach by peristaltic contractions of the esophageal wall. The esophagus is stripped clean of all bolus fluid by the contraction wave in normal swallowing. When the bolus fluid completely passes through the UES and UES closes, then the bolus is propelled downwards towards the stomach by a combination of gravitational forces and peristalsis.

Biofluids are propelled in tubes by transverse progressive waves propagating along the walls of the tubes in the peristalsis process. The flow of urine through the ureter, the movement of thyme in the entire gastrointestinal tract, and the transport of food-grains and liquids from the mouth through the esophagus are very common examples of this mechanism in the physiological world. Biomechanical pumps work in accordance with the same mechanism and are man-made instruments to pump physiological fluids, without hampering their purity. Misra and Pandey (2001) presented a mathematical model for the transport of food through the esophagus, by considering it to be governed by power-law.
Implementing the concept of inward contraction, study is made of sinusoidal wave propagation, restricted within the stationary wall of the esophagus, along its length.

Several investigations on the peristaltic transport through circular cylindrical tubes have been carried out by different researchers for Newtonian fluids (Brasseur et al., 1987; Shapiro et al., 1969; Takabatake et al., 1988; Yin and Fung, 1969) as well for non-Newtonian fluids (Bohme and Friedrich, 1983; Raju and Devanathan, 1972). Some studies on peristaltic transport through tubes of noncircular cross-sections are also available in the literature (Rao and Usha, 1995; Rath, 1982).

In all the studies mentioned earlier mentioned, the tube is considered to be of infinite length, and an assumption is made of infinite train-wave propagation. As a result of these considerations and assumptions, none of these studies are qualified to find applications to those physiological situations where a single wave travels down the length of an organ (for example, esophagus, and ureter) of finite dimension. $\mathrm{Li}$ and Brasseur (1993) pointed out this fact, and with the motive to explore the aspects of peristaltic pumping, they presented a model of peristaltic transport of a Newtonian viscous fluid, based on the classical lubrication theory, for arbitrary wave shape and arbitrary wave number through a finite length 
tube.

A finite-length single wave model of $\mathrm{Li}$ and Brasseur (1993) for Newtonian fluids is applicable only when the intake is water or some drink of similar physical properties. A bolus passing through the esophageal tube may be suitably modeled by considering a fluid of nonNewtonian nature. Carew and Pedley (1997) reported a peristaltic model with an active membrane through an infinite tube which remains occluded while relaxing, and activates only when it is subjected to a certain level of prestretch.

$\mathrm{Li}$ and Brasseur (1993) improved the conventional sinusoidal wave equation by considering the position of the wall a function of the minimum radius of the tube which vibrates in only one direction. The amplitude which is thus, the radius of the stationary tube minus the minimum tube-radius has to be adjusted whenever the degree of contraction of the tube is varied. The present authors, however, felt that since the wall fluctuates inwardly, it should be a function of the radius of the stationary tube rather than that of the contracted tube, and when the wall relaxes from contraction, the dilation should be restrained within the stationary boundary. This avoids the adjustment of the amplitude of the wave as required in the study of $\mathrm{Li}$ and Brasseur (1993) when the minimum radius is changed.

In this study, a new mathematical model is developed to calculate polynomial pressure distribution for the transport of food through the esophagus. The model is applied to a single contraction wave of normal swallowing in the esophagus. Analytical calculation from the new model on pressure distribution is in qualitative agreement with experimental observations.

\section{BIOLOGICAL DATA}

Manometry and videofluoroscopy are the two primary tools used to study esophageal motility, both in clinical and research setting. These two modalities provide fundamentally different information concerning bolus transport. Intraluminal manometry, which measures pressure at several fixed locations along the esophagus as a function of time, provides quantitative information regarding the forces applied to an ingested bolus by the esophageal musculature. The contraction wave is seen as a positive pressure on manometry which traverses the entire length of the esophageal body. On the other hand, videofluoroscopy records the geometry and motion of the esophageal wall in response to these forces.

The manometric evaluation of esophageal motility has focused on the peristaltic pressure waveforms that result from the aborad sequence of esophageal muscle contraction and relaxation. Brasseur et al. (1987) has studied interpretation of manometric data with and without concurrent radiographic image. An appreciation of mechanics of the swallowing process and bolus transport is necessary for proper interpretation of manometric intraluminal pressure measurements in terms of the spatial and temporal changes in lumen geometry.

Manometric pressure has been analyzed in the axial direction in order to describe the peristaltic pressure wave as it propagates through the esophagus in the direction of the bolus and to determine what sampling interval along the esophageal length is required for accurate representation. The appearance of the contraction wave as it propagates in the axial direction is important because of the interface of this wave with the bolus and additional appeal for understanding the coordination of underlying neuromuscular events responsible for transit (Clouse et al., 1996). Conventional manometric analysis found out that pressure distribution along the esophagus has a polynomial form. Ren et al. (1993) obtained this experimentally. The axial pressure profile within the bolus at a specific instant when the bolus was located in the distal esophagus is illustrated schematically in Figure 1.

Manometric analysis result of barium, wet and dry swallowing show that pressure distribution has a similar profile along the esophagus. This finding may attract attention that pressure distribution along the esophagus independent form bolus shape.

\section{Mathematical model}

Pressure distribution along the esophagus had been assumed sinusoidal in the literature, and as such, calculations have been done accordingly. However, experimental studies and biological data show that pressure distribution has a polynomial form. The goal is to develop an equation to calculate the spatial and temporal variations of intraluminal pressure $p(x, t)$ along the esophagus.

The esophagus is treated as a circular tube of finite length and flow is assumed with single-phase Newtonian incompressible fluid of uniform viscosity $\mu$. The characteristic velocity of the peristaltic wave is c, the wavelength of the bolus is $\lambda$, the tube length $L$, average radius of the bolus a and the minimum tube radius is $\varepsilon$. $A$ single contraction wave of peristaltic transport and pressure distribution are illustrated in figure 2. The fluid bolus is transported from left to right by contraction waves along the esophagus. The appropriate Reynolds number for peristaltic transport is:

$$
\operatorname{Re}=\frac{\rho c a}{\mu}\left(\frac{a}{\lambda}\right)
$$

The wall of the esophagus is contracted under the influence of a periodic transverse contraction wave which the passage is first shortened by way of contraction of 


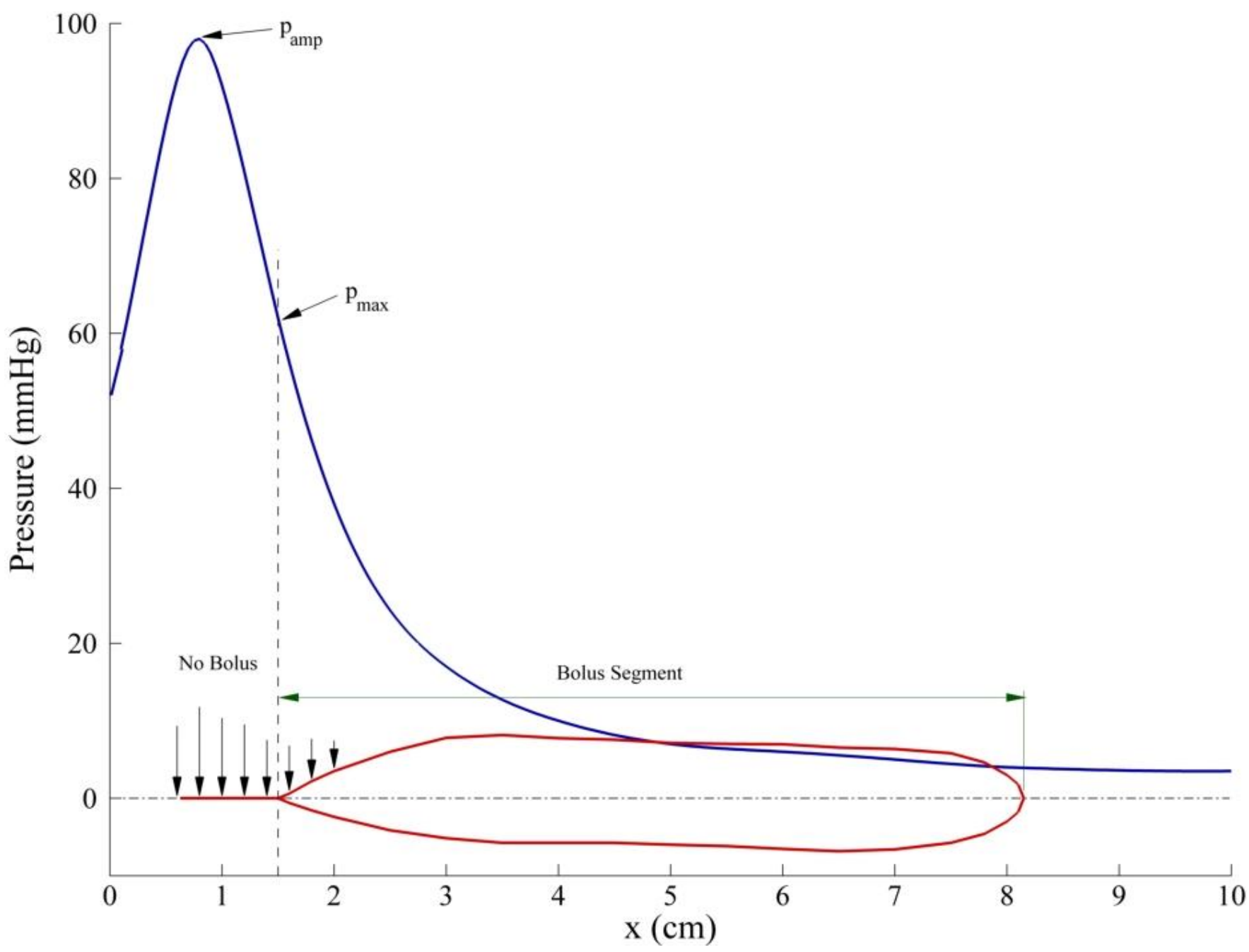

Figure 1. Axial distribution of intrabolus pressure during effective peristaltic transport of low viscosity barium bolus. Pressures had been measured during 2-7 swallows in 6 subjects at instant when proximal manometric site has determined on fluoroscopy to be 0.5 $\mathrm{cm}$ from tip of bolus tail. At this time bolus was in distal esophagus and esophageal emptying had not begun. Seven manometric sites were spaced $1 \mathrm{~cm}$ apart. Vertical dashed line indicates boundary between bolus-containing and lumen-occluded esophageal segments and is theoretically site of maximum intrabolus pressure $\left(\mathrm{p}_{\max }\right)$. Intrabolus pressure, located in region of bolus tail that is contiguous to segment of actively contracting esophageal wall (vertical arrows), was significantly greater $(p<0.05)$ than that at other sites, which were not significantly different from each other (Ren et al., 1993).

muscles. Then, its path is retracted so that its original position is attained. This process continues until the bolus is completely squeezed out. The shape of the bolus tail and head is described using sinusoidal functions, and the main body of the bolus is approximated with a constant radius. Mathematically, it can be expressed as follows:

$$
H(x, t)=\varepsilon+0.5 \alpha\left(1-\cos 2 \pi\left(\frac{x-c t}{\lambda}\right)\right)
$$

Here, $\alpha$ is the wave amplitude and $\alpha=2(a-\varepsilon)$.

Intraluminal pressure in a moving bolus has the force applied to the bolus at the esophageal wall. This applied pressure may be due to passive elasticity in the esophageal wall, or active muscle tension, primarily in the circular muscle. However, intrabolus pressure is not directly related to the wall tension. When there is significant motion, part of the tensile force within the esophageal wall goes into accelerating the wall and the fluid (Brasseur and Dodds, 1991). It should be considered that pressure wave, as seen in Figure 2, covers the entire esophagus which begins at the UES and ends at the LES at any specific time. Pressure distribution along the esophagus can be defined as:

1. No-bolus region: high pressure zone $\left(p_{0}+p_{1}\right)$,

2. Bolus segment: pressure gradient decrease gradually, 


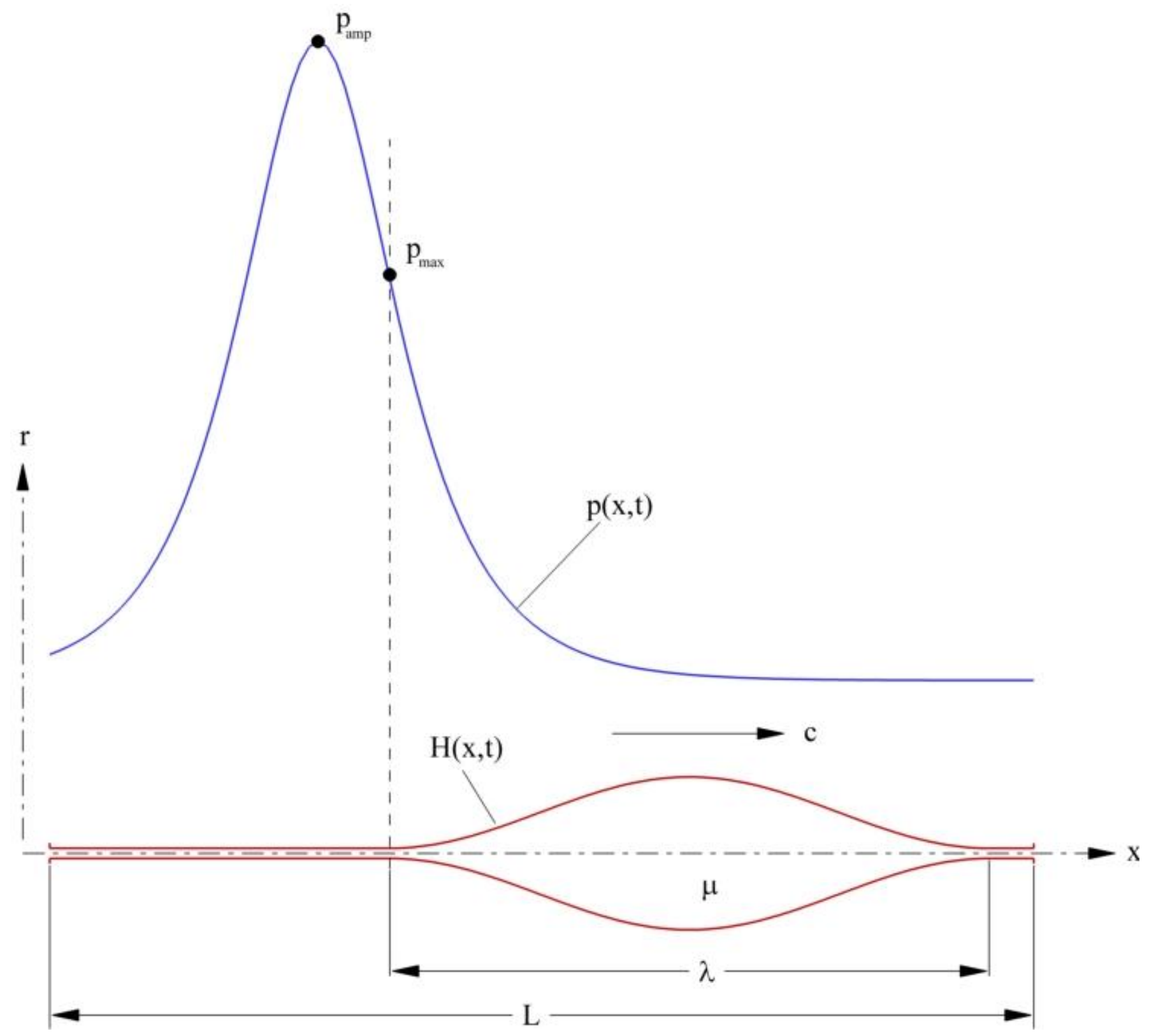

Figure 2. Schematic representation of Bolus geometry, pressure distribution, coordinates system and nomenclature of the model.

$p_{\max }$ is effected at the bolus tail $\left(p_{0}+p_{1}\right)$,

3. After bolus segment: low pressure zone $\left(p_{0}\right)$. Pressure wave travels along the esophagus in time. $\quad \frac{\partial p}{\partial x}=-p_{1} \sec h\left(\frac{b_{1}(x-c t)-b_{2}}{L}\right) \tanh \left(\frac{b_{1}(x-c t)-b_{2}}{L}\right)$
These approaches can be expressed mathematically as:

$p(x, t)=p_{0}+p_{1} \operatorname{sech}\left(\frac{b_{1}(x-c t)-b_{2}}{L}\right)$

Here, $b_{1}, b_{2}$ and $b_{3}$ are constants; $p_{0}$ and $p_{1}$ are constants of resting and active pressures.
The first derivative of governing equation is as follow:

The equation of conservation of momentum in an axisymmetric coordinate system moving with the wave shape at speed $c$ is written in the literature as follow:

$\frac{\partial p}{\partial x}=\frac{\mu}{r} \frac{\partial}{\partial r}\left(r \frac{\partial u}{\partial r}\right)$ 
Table 1. Values of coefficients

\begin{tabular}{ccc}
\hline Symbol & Value & Unit \\
\hline$p_{0}$ & 3.5 & $\mathrm{mmHg}$ \\
$p_{1}$ & 94.5 & $\mathrm{mmHg}$ \\
$b_{1}$ & 14 & - \\
$b_{2}$ & 11 & - \\
\hline
\end{tabular}

$\frac{\partial p}{\partial r}=0$

The axial and radial velocities:

$$
\begin{aligned}
& u=\frac{1}{4 \mu} \frac{\partial p}{\partial x}\left(r^{2}-H^{2}\right) \\
& v=\frac{r}{4 \mu}\left[H \frac{\partial H}{\partial x} \frac{\partial p}{\partial x}-\frac{\partial^{2} p}{\partial x^{2}}\left(\frac{1}{4} r^{2}-\frac{1}{2} H^{2}\right)\right]
\end{aligned}
$$

Equation 3 is the governing equation for the pressure distribution along the entire esophagus. However, equations 5, 6, 7 and 8 have been applied on the bolus segment.

\section{Numerical method}

The numerical method to solve the equation of pressure is based on predictor-corrector approximation method. Pressure in Figure 1 is experimentally obtained from manometric analysis in the literature. This is denoted as $p_{e}(x, 0)$ at $t=0 \mathrm{~s}$ and $L=10 \mathrm{~cm}$. To calculate pressure distribution $(p(x, t))$ from equation (3), $b_{1}, b_{2}, p_{0}$ and $p_{1}$ should be known. Equation (3) can be rewritten as follow:

$$
p\left(x_{i}, t_{j}\right)=p_{0_{k}}+p_{1_{k}} \operatorname{sech}\left(\frac{b_{1_{k}}\left(x_{i}-c t_{j}\right)-b_{2_{k}}}{L}\right)
$$

\section{Step 1: Selection of initial values}

$$
\begin{aligned}
& \text { Experimentally } \quad \text { found } \\
& \text { fort }=0 ; p_{e}\left(x_{i}, 0\right)=p\left(x_{i}, 0\right) \\
& b_{1}=1 \\
& b_{2}=0 \\
& p_{0}=0
\end{aligned}
$$

Equation (9) is rearranged to calculate the initial value of $p_{1}$ as:

$$
p_{1}=\frac{p\left(x_{i}, 0\right)}{\operatorname{sech}\left(\frac{x_{i}}{L}\right)}
$$

\section{Step 2: Calculation of $p\left(x_{\tilde{i}}, 0\right)$}

Selected initial values of coefficients are substituted in equation (9), and $p\left(x_{i}, 0\right)$ are calculated.

\section{Step 3: Relative error of approximation}

Relative error $(\eta)$ of approximation is found by applying equation (10). Note that the relative error is unrelated to convergence in the algorithm but instead to the step size and approximation of the actual function. The step size is selected relatively small in order to get a good approximation.

$\eta_{x_{i}}=\frac{\left|p\left(x_{i}, 0\right)-p_{e}\left(x_{i}, 0\right)\right|}{\left|p\left(x_{i}, 0\right)\right|}$

Iteration process is repeated until the guesses converge within desired relative error. Finally, the best approximation is obtained after 50 iterations and the values in Table 1 are obtained. Equation (3) is rearranged by replacing coefficients with numerical values and analytical calculation is done; so, analytical pressure is calculated.

Analytical and experimental pressure distributions along the esophagus are illustrated with analytical and experimental boluses (wall shapes) in Figure 3. Bolus can be already calculated analytically in the literature. However, analytical calculation of polynomial pressure form could not be found in the literature. Findings of our new model show that polynomial form of pressure distribution along the esophagus can be calculated successfully. Schematic comparisons of analytical and experimental findings (Figure 3) show that results are overlapped in almost best fit. Relative error may be seen in Figure 4 with error distribution along the esophagus ( $x$ direction).

\section{Pressure distribution}

Normal esophageal peristalsis involves a single wave of active muscle contraction preceded by a single wave of muscle relaxation; therefore, the bolus is isolated within a single peristaltic wave. The dominant characteristic of bolus transport is apparently seen in Figure 1 as the complete stripping of the bolus from the esophageal lumen by a progressive contraction wave of sufficient force to maintain closure in the tail segment as it passes along the esophagus. To model this primary transport characteristic of esophageal musculature, it is considered that an idealized peristaltic wave in which the shape of the tail region and the wave speed are not varied as the contraction wave passes along the esophageal lumen.

A single peristaltic contraction wave which is traveling along the esophagus is illustrated in Figure 5. Detailed variations in pressure amplitude are not modeled. At Figure $3(\mathrm{a}), \mathrm{t}=0 \mathrm{~s}$, pressure distribution and single bolus 


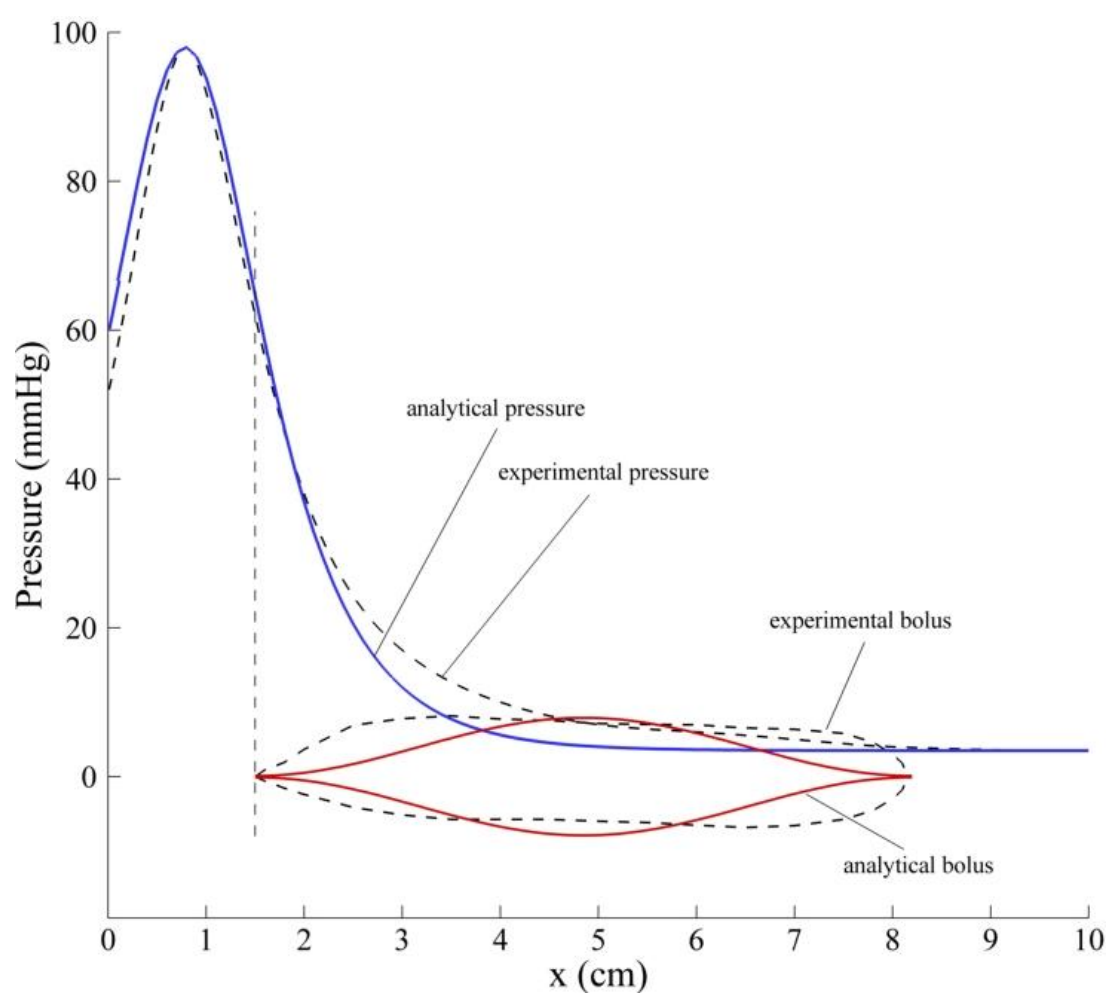

Figure 3. Schematic illustrations of computed (analytical) pressure distributions from new model, analytical bolus (wall shape) from equation (2), experimental pressure distributions and bolus (wall shape) from Ren at al., (1993).

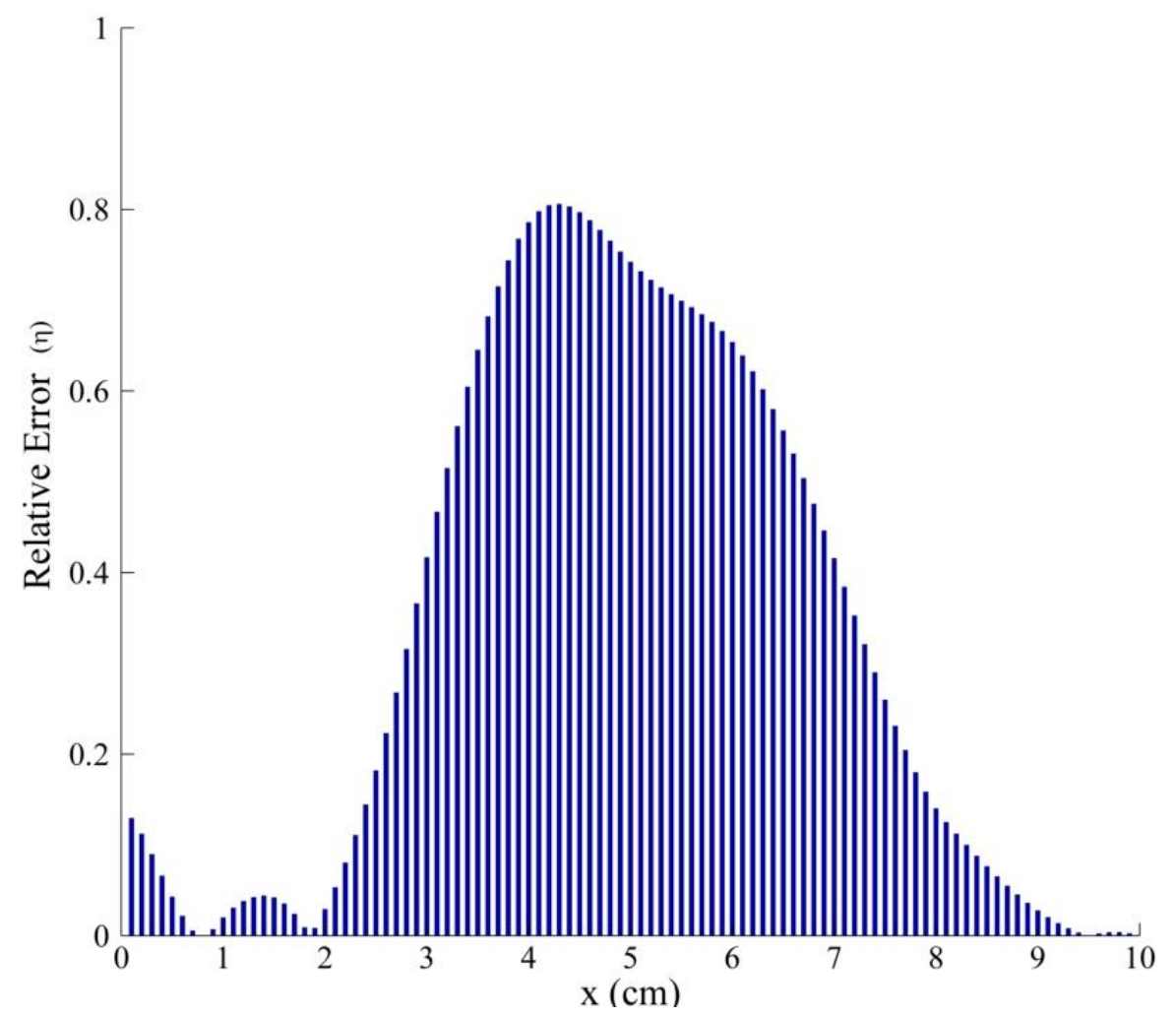

Figure 4. Relative error ( $\eta)$. 

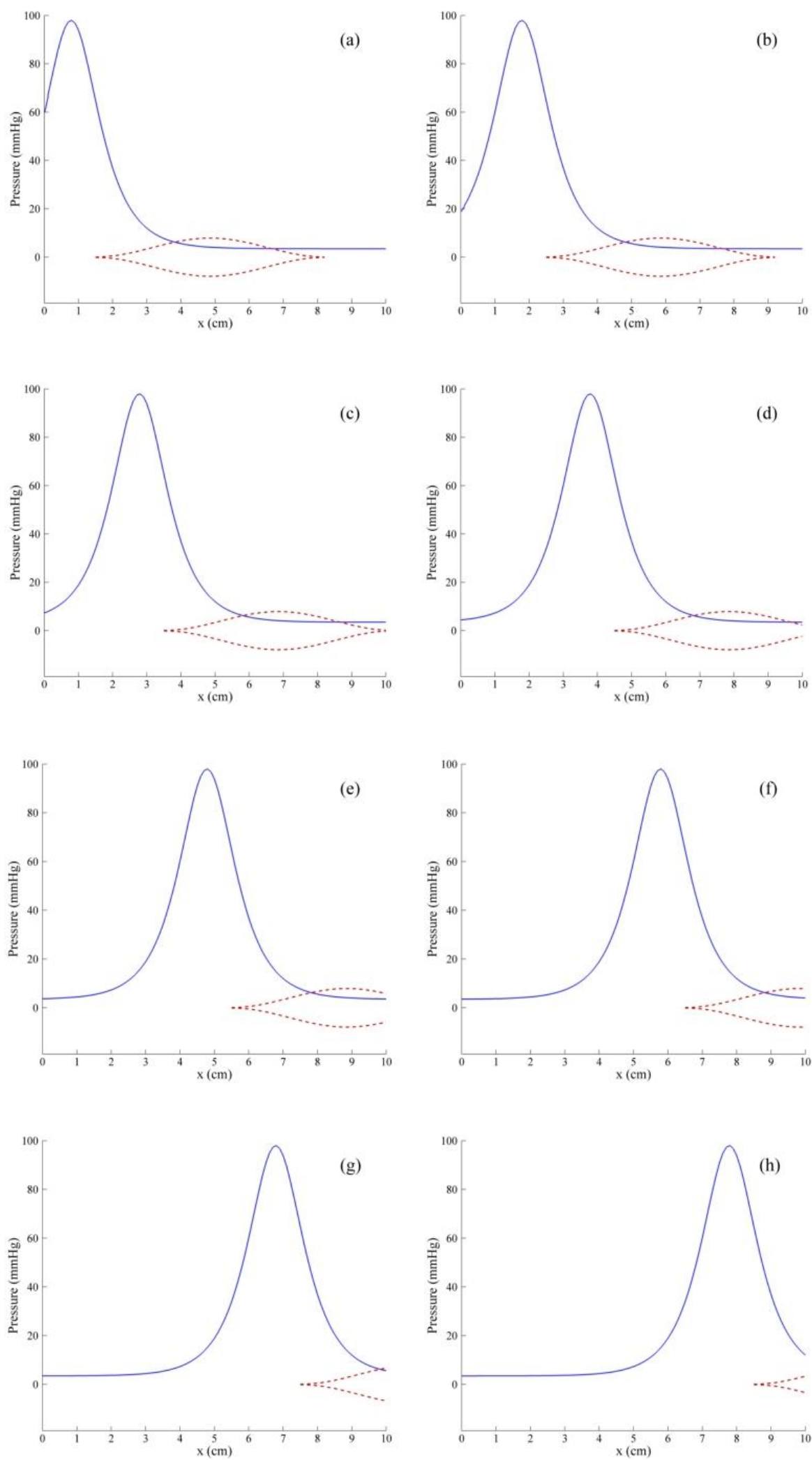

Figure 5. Pressure distribution along the esophagus (tube) at eight time instants in one wave period with one wave in the esophagus (tube). (a) $t=0 \mathrm{~s}$; (b) $t=0.5 \mathrm{~s}$, (c) $t=1 \mathrm{~s}$, (d) $t=1.5 \mathrm{~s}$, (e) $t=2 \mathrm{~s}$, (f) $\mathrm{t}=2.5 \mathrm{~s},(\mathrm{~g}) \mathrm{t}=3 \mathrm{~s}$, (h) $\mathrm{t}=3,5 \mathrm{~s}$. The solid lines are pressure distributions along the esophagus (tube), and the dashed lines are the corresponding boluses (wall shapes). The wave travels with wave speed, $2 \mathrm{~cm} / \mathrm{s}$, from left to right. 


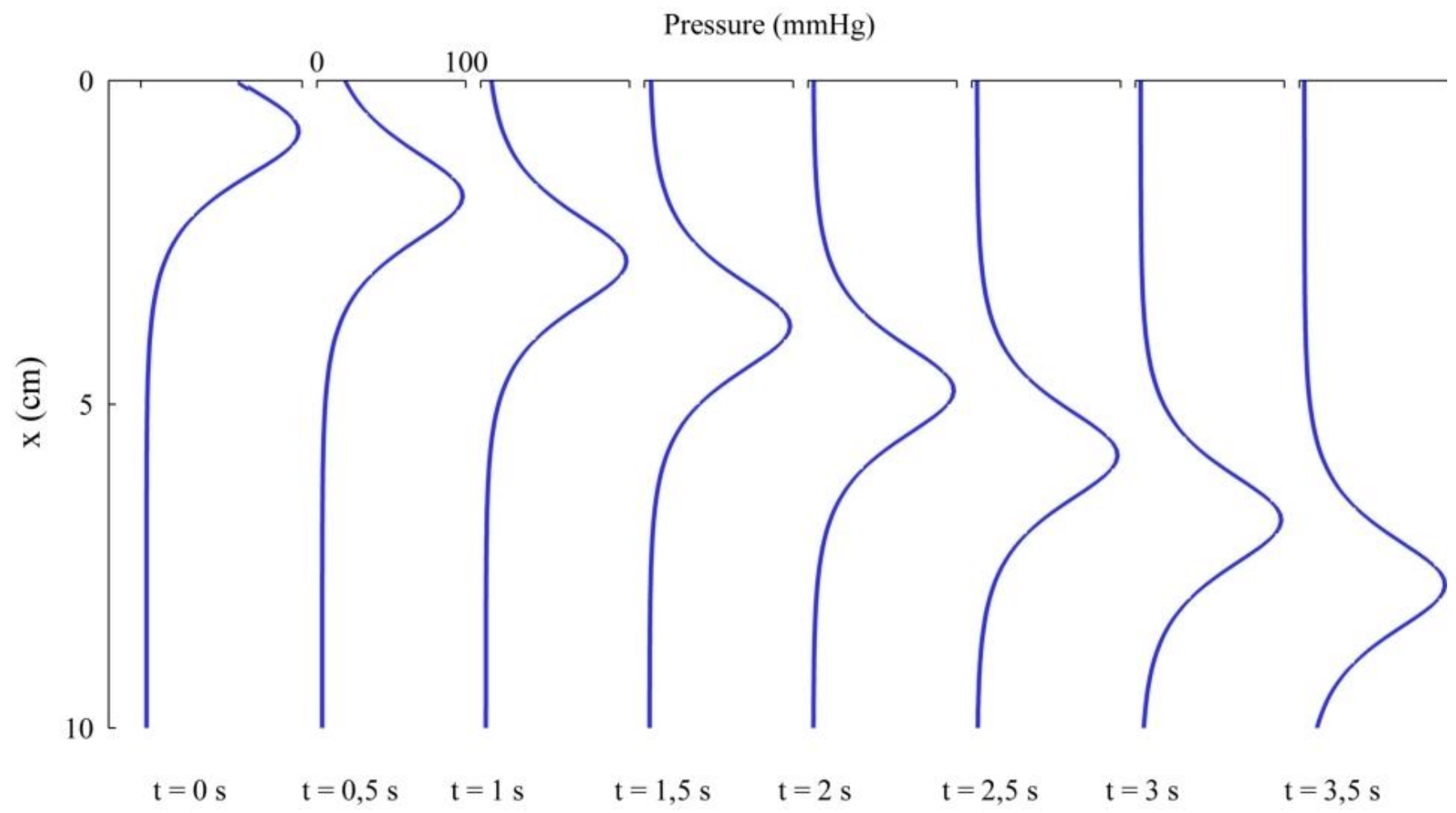

Figure 6. Spatial and temporal illustrations of pressure distribution.

are seen, and it travels with wave speed $2 \mathrm{~cm} / \mathrm{s}$ from left to right and movement of both pressure wave and bolus are also observed in Figures 5b, c, d, e, f, $g$ and $h$. Spatial and temporal variation of pressure distribution is illustrated in Figure 6.

The methodology of this study involves combining mathematical modeling with biological data. The concept is to embed the essential dynamical principles required to properly characterize esophageal bolus transport into a mathematical model from which intraluminal geometry variations may be directly related to intraluminal pressure variations and then to develop mathematical model to capture the global characteristics of videofluoroscopic and concurrent manometric data from specific case studies. The global characteristics of a specific esophageal study are properly calculated (Figures 5 and 6 ), the temporal variations in the intraluminal geometry pressure relationship can be obtained in detail.

\section{CONCLUSIONS}

The mechanics of the swallowing process and bolus transport was needed for proper mathematical model to calculate global variations. So, a new mathematical model is developed to successfully calculate pressure distribution along the esophagus. The model is applied to a single contraction wave of normal swallowing in the esophagus. Analytical calculation from the new model on pressure distribution is in qualitative agreement with experimental observations. The crucial conclusions to draw from results are that this model is qualified to calculate some biological situations where a single wave travels down the length of an organ (for example, esophagus, and ureter) of finite dimension. On the other hand, it is going to help to develop more realistic mathematical expression to explain other variations of peristaltic transport in the esophagus and ureter.

\section{REFERENCES}

Bohme GB, Friedrich R (1983). Peristaltic Flow of Viscoelastic Liquids. J. Fluid Mech., 128: 109-122.

Brasseur JG, Corrsin S, Lu NQ (1987). The Influence of a Peripheral Layer of Different Viscosity on Peristaltic Pumping with Newtonian Fluids. J. Fluid Mech., 174: 495-519.

Brasseur JG, Dodds WJ (1991). Interpretation of Intraluminal Manometric Measurements in Terms of Swallowing Mechanics. Dysphagia, 6: 100-119.

Carew EO, Pedley TJ (1997). An Active Membrane Model for Peristaltic Pumping: Part 1 - Periodic Activation Waves in an Infinite Tube. Trans. ASME J. Biomech. Engng. 119: 66-76.

Clouse RE, Stanio A, Bickston SJ, Cohn SM (1996). Characteristics of Propagating Pressure Wave in the Esophagus. Dig. Dis. Sci., 41(12): 2369-2376.

Li M, Brasseur JG (1993). Non-Steady Peristaltic Transport in FiniteLength Tubes. J. Fluid Mech., 248: 129-151.

Misra JC, Pandey SK (2001). A Mathematical Model for Oesophageal Swallowing of a Food Bolus. Math. Comput. Model., 33: 997-1009. 
Raju KK, Devanathan R (1972). Peristaltic Motion of a Non-Newtonian Fluid. Rheol. Acta., 11: 170-178.

Rao AR, Usha S (1995). Peristaltic Transport of Two Immiscible Viscous Fluids in a Circular Cylindrical Tube. J. Fluid Mech., 298: 271-285.

Ren J, Massey BT, Dodds WJ, Kern MJ, Brasseur JG, Shaker R, Harrington SS, Hogan WJ, Arndorfer RC (1993). Determinants of Intrabolus Pressure During Esophageal Peristaltic Transport. Am J Physiol., 264: G407-G413.

Shapiro AH, Jaffrin MY, Weinberg SL (1969). Peristaltic Pumping with Long Wave-Lengths at Low Reynolds Number. J. Fluid Mech., 37: 799-825.
Takabatake S, Ayukawa K, Mori A (1988). Peristaltic Pumping in Circular Cylindrical Tubes: A Numerical Study of Fluid Transport and its Efficiency. J.Fluid Mech., 193: 267-283.

Rath HJ (1982). Peristaltic Flow Through a Lobe-Shaped Tube. Intl. J. Mech Sci., 24: 359-367.

Yin F, Fung YC (1969). Peristaltic Waves in a Circular Cylindrical Tube. J. Applied Mech., 36: 93-112. 